\title{
Zur Kenntnis afrikanischer und orientalischer Culiciden.
}

\section{Von Dr. Günther Enderlein,}

Verwalter der Centralstelle für blutsaugende lnsekten am Zoologischen Museum der Universität Berlin.

Mit 2 Abbilduugen im Text.

Nachstehend werden einige neue Culiciden bekannt gemacht, sowie einige Fundorte von bekannten Arten gegeben. Das Material stammt aus den Ausbeuten von Professor Dr. Ziemann, Professor Dr. Voeltzk ow und anderen.

Berlin, 24. 1. 1920.

Lepiothauma nov. gen.

Typus: L. furfurea nov. spec.

(Fig. 1 u. 2.)

Die Unterschiede von Mucidus Theob. 1901 sind:

Die Gabelzelle $\mathrm{m}_{2}+\mathrm{m}_{3}$ reicht weiter proximalwärts als die Gabelzelle des Radialramus $\left(\mathrm{r}_{\mathrm{s}}+_{3}+\mathrm{r}_{4}+_{5}\right)$. Beide Gabelzellen 'sind daher lang, die vordere ist aber nicht verkürzt. Schenkel ziemlich stark seitlich zusammengedrückt, besonders die mittleren und hinteren. End-

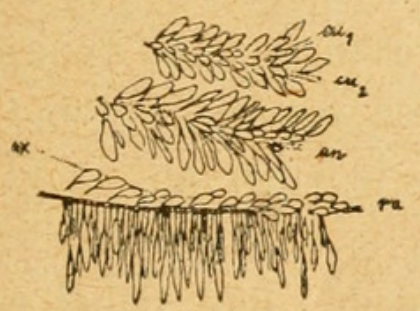

Fig. 1. Flügelstück vom Hinterrand mit den Schuppen. $r a=$ Rand. Vergr. ca. $25: 1$.

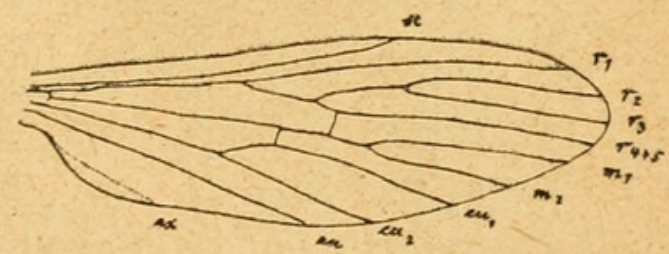

Fig. 2. Lepiothauma furfurea Enderl. Flügelgeäder. Vergr. $12: 1$

viertel aller Schenkel und Basalviertel aller Schienen mit langgestielten, abstehenden, breiten Schuppen besetzt. Flügeladern mit sehr breiten, spindelförmigen Schuppen besetzt. Flügelrand mit sehr langen, schmalen, spindelförmigen Schuppen besetzt. Axillarader nackt. Flügelrand am Hinterrand der Axillarzelle und anschliessend bis an die Mündung der Analis nach auswärts noch mit breiten, spindelförmigen Schuppen besetzt. Flügelschuppen breit, häufig am Ende mehr oder weniger abgeschrägt. 


\section{Lepiothauma furfurea nov. spec.}

o Kopf schwarzbraun, die braunen Schuppen sind mit weissen untermischt. Fühler dunkelbraun. Rüssel schlank, schwarz, in der Mitte ein weisser Ring, der doppelt so lang als die Rüsseldicke ist, Endviertel oben mit weissen Schuppen untermischt. Palpen ca. ${ }^{1 / 4}$ der Rüssellänge, schwarz, die beiden letzten Glieder am Ende mit einigen weissen Schuppen. Thorax hellbraun mit weissgrauen Schuppen. Scutellum mit breiten weissen Schuppen, die Mitte der 3 Lobi mit einigen breiten schwarzen Schuppen. Abdomen hell braungelb, mit ebensolchen Schuppen besetzt, die nur mit einzelnen weisslichen untermischt sind. Beine hell gelbbraun. Schenkel mit hellbraunen Schuppen, vorn am Ober- und Unterrande mit einer Reihe kleiner Fleeke aus weissen Schuppen; je ein oberer Fleck steht iiber einem unteren. Der Hinterschenkel trägt 7 solcher Fleckpaare, ausserdem am Ende einen kleinen gelblichweissen Fleck; Schienen mit hellbraunen Schuppen, oben eine Längsseite Querstriche aus weissen Schuppen, die Hinterschiene trägt 7 solcher Querstriche, den einen davon am Ende. Tarsen hell graubraun, 2. und 3. Vordertarsenglied eir wenig heller an der Basis, Basalviertel des 2. und 4. und Basalhälfte des 3. Mitteltarsengliedes weiss, 1 . Hintertarsenglied oben mit 4 weissen Fleckchen, eins an der Basis, eins am Ende des 1. Drittels, eins am Ende des 2. Drittels und eins an der Spitze; die übrigen Tarsenglieder abgebrochen. Flügel hyalin, dicht mit grossen braunen Schuppen besetzt, dazwischen ganz vereinzelte weisse Schuppen eingestreut und mit einem grösseren quadratischen weissen Schuppenfleck am Ende von sc vom Rande bis auf $r_{1}$ sich erstreckend und mit folgenden kleineren weissen Schuppenflecken: je einer am Ende von $r_{1}$, $r_{2}, r_{3}, r_{4}+{ }_{5}, m_{1}, m_{2}, c u_{1}$ und an, je ein etwas grösserer an den Gabelungspunkten der Cubitalgabel, Mediangabel und Gabel $r_{2}$ und $r_{3}$. Weisse Schuppen finden sich terner reichlich an der Flügelbasis.

Körperlänge $4^{1 / 4} \mathrm{~mm}$. Flügellänge $3,2 \mathrm{~mm}$. Rüssellänge $1,6 \mathrm{~mm}$.

Westafrika, im Innern von Kamerun, Mao Godi. 11. Juni 1909. 1 ㅇ gesammelt von Riggenbach.

Aniella nov. gen.

Typus: A. africana (Theob. 1901).

Die Augen stossen so dicht zusammmen, dass keine Schuppen zwischen ihnen inserieren können. Stegomyia Theob. 1901 unterscheidet sich von dieser Gattung dadurch, dass zwischen den Augen ein 
schmaler Streifen Stirn übrig ist, auf dem zahlreiche silberweisse Schuppen fussen.

Ob hier noch einzuordnen ist: St. pollinctor Grah. 1910 (Westafrika) und St. apiceargentea Theob. 1910 (Afrika) ist nicht sicher.

Aniella africana (Theob. 1901).

Kamerun, Duala. 4. Okt 1906. 1 \& gesammelt von Professor Dr. Zi e m a n n.

Kamerun. Juni 1903. 1 \& gesammelt von Professor Dr. $\mathrm{Zi} \mathrm{e} m$ ann.

Kamerun, Duala-Hafen. 18. Juli 1901. 1 f gesammelt von Dr. Zu pitza.

Togo, Bismarckburg. 20. 7. - 20. 9. 1890. 1 우 gesammelt von $R$. B ütt n e r.

Aniella longipalpis (Grünb. 1905).

Westafrika. 3 ㅇ gesammelt von Professor. Dr. Zi emann.

\section{Aniella Ziemanni nov. spec.}

\& Kopf dunkelbraun; silberweisse Schuppen finden sich an folgenden Stellen: die Oberseite und Spitze des Palpenendgliedes, ein schmaler Schläfensaum und ein breiter medianer Längsstreifen auf dem Scheitel. Thorax dunkelbraun; silberweise l'ärbung der Schuppen ist an folgenden Stellen: auf dem Rückenschild vorn ein kurzer medianer Längsstreif und in der Mitte der vorderen Hälfte jederseits ein grosser rundlicher Fleck von ca. $0,3 \mathrm{~mm}$ Durchmesser, auf dem Scutellum, einige Flecken auf den Pleuren und je ein Fleck auf der Aussenseite der Coxen. Abdomen ähnlich wie bei St. fasciata, nur erstrecken sich die silberweissen Schuppen auf deın 5., 6. und 7. Tergit über die ganze Oberfläche; ob dies auch bei den übrigen Tergiten der Fall ist, kann nicht festgestellt werden, da stark abgerieben; doch steht auf der Mitte des 3. Tergites eine Anzahl silberweisser Schuppen. 1) ie silberweissen Schuppen nehmen auf den Schenkeln fast das Endsechstel ein. Beine dunkellraun. Basis der Vorderschiene gelblich; Unterseite des 2. Viertels der Hinterschiene silberweiss. Vordertarsus: Basalachtel des 1. Gliedes, Basalviertel des 2. Gliedes weiss, Unterseite des 2., 3. und 4. Gliedes etwas weisslich. Mitteltarsus: Basalsechstel des 1. Gliedes und das 2. Glied ohne die äusserste Spitze weiss, 3.-5. Glied dunkelbraun. Hintertarsus: Basalviertel des 1. 2. und 3. Gliedes weiss, 4. Glied dunkelbraun (5. Glied abgebrochen). Klauen der Vorder- und Mittelbeine kurz gezähnt. Flügelschuppen braun. 
Körperlänge $4^{3} / 4 \mathrm{~mm}$. Rüssellänge $1^{3 / 4} \mathrm{~mm}$. Flügellänge $3 \frac{1}{2} \mathrm{~mm}$.

$\mathrm{S} u ̈ d-K a m e r u n$. (Bei Kribi oder bei Wuri). 1 의 gesammelt von Professor Dr. Zi emann.

Gewidmet sei diese auffällige und schön gezeichnete Art dem Sammler.

Am nächsten steht ihr A. nigeria (Theob. 1901) und A. sugens (Wied 1828).

\section{Aniella togoensis nov. spec.}

7. Die Unterschiede von A. Ziemanni sind folgende:

Spitze des Rüssels grauweisslich. Schenkel dunkelbraun, Mittelschenkel aussen in der Mitte und am Ende mit silberweissem Fleck, Hinterschenkel aussen mit silberweisser Längslinie, die eine Spur bläulichen Ton besitzt, Mittelschiene einfarbig dunkelbraun, Hinterschiene auf der Unterseite der Basis gelblich, auf der Aussenseite des 2. Viertels silberweiss. Mitteltarsus: Basalsechstel des 1. Gliedes und das 2. Glied mit Ausnahme eines blassbräunlichen Streifes auf der Unterseite weiss (vom 3. Glied ab abgebrochen). Hintertarsus: Basalfünftel des 1. und 2. Gliedes, Basalsechstel des 3. Gliedes und das 4. Glied mit Ausnahme der äussersten Spitze weiss; 5. Glied dunkelbraun. Basalhälften des 5., 6. und 7. Abdominaltergites silberweiss, Seiten aller Tergite mit grossem, bläulich silberweissem Fleck. Tergite sonst dunkelbraun.

Körperlänge $3 \mathrm{~mm}$. Flügellänge $2^{1 / 2} \mathrm{~mm}$. Rüssellänge $1,5 \mathrm{~mm}$.

Westafrika, Togo. Klein Popo. Juni 1900. 1 \& gesammelt von Professor Dr. Z i e mann.

Aniella argenteopunctata (Theob. 1901).

Deutsch-Ost-Afrika, Bukoba. 13 우 gesammelt von $\mathrm{H} \ddot{\text { s semann. }}$

\section{Phagomyia Theob. 1905.}

Phagomyia nigricephala (Theob. 1901).

K a merun, Mai 1903, 1 ; ; November 1903, 1 ㅇ gesammelt von Professor Dr. Zi emann.

Phagomyia irritans (Theob. 1901).

Deutsch-Ost-Afrika, Kilwa. 26. Nov. 1906. 1 와 gesammelt von $H \ddot{\text { semann}}$.

Phagomyia mutica nov. spec.

9. Diese Spezies stimmt mit P. nigricephala (Theob.) auffällig überein und unterscheidet. sich nur dadurch, dass die Klauen aller 
Füsse einfach sind, während bei $P$. nigricepluala die Klauen der Vorder- und Mittelfüsse mit einem langen mittleren Seitenzahn versehen sind.

Britisch-0st-Afrika, Insel Patta. 16. Febr, 1903. 4 우우 yesammelt von Professor Dr. Voeltzkow.

Theobaldia Nev.-Lem. 1902.

Theobaldia spathipalpis (Rond. 1872).

S. -W.-Afrika. Rooibank. Mai 1905. $2 \sigma^{\top} \sigma^{\top} 2$ 우 우 gesammelt von Professor Dr. L. S chultze.

Mansonia Blanch. 1901.

Mansonia annulipes (Wálk. 1857).

Pandjang-Goenoeng. 1 \& (durch Professor Dr. Dönitz).

Anophelinae.

Myzorhynchus Blanch. 1902.

Myzorhynchus sinensis (Wied 1828).

Formosa. Takao. Mai 1907. 19 우우

H. Sauter.

Anping. 30. Nov. 1906. 1 \& gesammelt von

Japan. Jokkaichi, im Zimmer. 24. Febr. 1906. 1 ㅇ gesammelt von H. Sauter.

Pyretophorus Blanch. 1902.

Pyretophorus cinereus (Theob. 1901).

Süd-West-Afrika, Rooi - Bank. Mai 1905. 2 우 gesammelt von Professor Dr. Schultze.

West-Afrika, Togo, Kete Kratje. Jan. 1900. 1 \& gesammelt von Graf $\mathrm{Zech}$.

\section{Megarhininae.}

Toxorhynchites Theob. 1901.

Toxorhynchites Gilesi Theob. 1901.

Nord-Indien, Sikkim. $1 \sigma^{\top}$ gesammelt von Bingham.

China, Canton. 1 ?.

Die Art war bisher nur aus Vorder- und Hinter-Indien, Assam und Ceylon bekannt. 


\section{$2 \mathrm{BHL}$ Biodiversity Heritage Library}

Enderlein, Günther. 1923. "Zur Kenntnis afrikanischer und orientalischer Culiciden." Wiener entomologische Zeitung 40, 25-29. https://doi.org/10.5962/bhl.part.2580.

View This Item Online: $\underline{\text { https://www.biodiversitylibrary.org/item/43768 }}$

DOI: https://doi.org/10.5962/bhl.part.2580

Permalink: https://www.biodiversitylibrary.org/partpdf/2580

\section{Holding Institution}

Smithsonian Libraries

\section{Sponsored by}

Smithsonian

\section{Copyright \& Reuse}

Copyright Status: NOT_IN_COPYRIGHT

This document was created from content at the Biodiversity Heritage Library, the world's largest open access digital library for biodiversity literature and archives. Visit BHL at https://www.biodiversitylibrary.org. 